\title{
A Very Tight Approximation of the SISO Energy Efficiency-Spectral Efficiency Trade-Off
}

\author{
Fabien Héliot, Member, IEEE, Muhammad Ali Imran, Member, IEEE, and Rahim Tafazolli, Senior Member, IEEE
}

\begin{abstract}
Beside the well-established spectral-efficiency (SE), energy-efficiency (EE) is currently becoming an important performance evaluation metric, which in turn makes the EE-SE trade-off as a prominent criterion for efficiently designing future communication systems. In this letter, we propose a very tight closed-form approximation (CFA) of this trade-off over the singleinput single-output (SISO) Rayleigh flat fading channel. We first derive an improved approximation of the SISO ergodic capacity by means of a parametric function and then utilize it for obtaining our novel EE-SE trade-off CFA, which is also generalized for the symmetric multi-input multi-output channel. We compare our CFA with existing CFAs and show its improved accuracy in comparison with the latter.
\end{abstract}

Index Terms-Energy-efficiency, spectral-efficiency, trade-off, single-input single-output, Rayleigh fading, closed-form.

\section{INTRODUCTION}

The current rise in energy demand and price will soon make energy as valuable as spectrum for communication network operators, which explains the recent trend towards more energy-efficient communication networks [1]. The efficiency of communication systems has usually been assessed via the $\mathrm{bit} / \mathrm{s} / \mathrm{Hz}$ metric, which indicates how efficiently a limited frequency spectrum is used but fails to indicate how efficiently energy is consumed. As the latter is becoming as important as the former, energy-efficiency (EE) or consumption metric such as the bit/J [2] or J/bit [2], [3], respectively, must also be included in the performance evaluation framework.

Maximizing the EE, or equivalently minimizing the consumed energy, while maximizing the spectral-efficiency (SE) are conflicting objectives which implies the existence of a trade-off. The EE-SE trade-off concept has first been introduced in [3], where an approximation of this trade-off has been derived for the white and colored noise, as well as multiinput multi-output (MIMO) fading channels. We have recently proposed in [4], a simple and accurate closed-form approximation (CFA) of this EE-SE trade-off for the MIMO Rayleigh flat fading channel. Single-input single-output (SISO) channel being a special case of MIMO, the approximation method of [3] and our CFA in [4] can also be applied to the SISO scenario. However, both these CFAs are mainly accurate at low SE in the SISO case. Thus, there is a need for designing a dedicated and accurate CFA of the SISO EE-SE trade-off, as we have already attempted in [5].

In this letter, our contributions are three-fold:

F. Héliot, M.A. Imran, and R. Tafazolli are with the Centre for Communication Systems Research, FEPS, University of Surrey, Guildford GU2 7XH, UK (phone: +44 1483689 492; fax: +44 1483686 011; E-mail: F.Heliot@Surrey.ac.uk). The research leading to these results has received funding from the European Commission's Seventh Framework Programme FP7/2007-2013 under grant agreement $n^{\circ} 247733$-project EARTH.
- The derivation of a very accurate CFA of the ergodic capacity over the SISO Rayleigh flat fading channel, which differs at most by $0.5 \%$ in comparison with the exact SISO Rayleigh flat fading ergodic capacity.

- The derivation of a very tight CFA of the SISO EE-SE trade-off, which differs at most by $0.05 \mathrm{~dB}$ in comparison with the nearly-exact SISO EE-SE trade-off.

- The generalization of this CFA for the symmetric MIMO Rayleigh flat fading channel.

The rest of the letter is organized as follows, Section II introduces the EE-SE trade-off concept and the approximation method of [3]. In Section III, we derive an improved CFA of the SISO ergodic capacity by means of a parametric function. We then use this expression in Section IV for designing our very tight CFA of the SISO EE-SE trade-off, which is also generalized for the symmetric MIMO case. Numerical results show the great accuracy of our novel CFA of the EESE trade-off for both SISO and symmetric MIMO channels in comparison with existing ones. Conclusions are drawn in Section V. A preliminary version of this work can be found in [5], which has been significantly improved in this paper both in terms of accuracy and simplicity of formulation.

\section{EE-SE TRADE-OFF}

The EE-SE trade-off concept can simply be described as how to express EE as a function of SE. Let us assume a communication system consuming a total power of $P_{\Sigma}$ Watt for achieving a transmission rate of $R$ bit/s, then its energy consumption per bit can be defined as $E_{b}=P_{\Sigma} / R$ and its EE-SE trade-off can be formulated as [3]

$$
\frac{E_{b}}{N_{0}}=\frac{f^{-1}(C)}{S},
$$

when assuming an idealistic power model, i.e. the total consumed power $P_{\Sigma}$ is equal to the transmit power $P$, and where

$$
C=f(\gamma) \quad(\mathrm{bit} / \mathrm{s} / \mathrm{Hz})
$$

is the channel capacity per unit bandwidth, $\gamma=P /\left(N_{0} W\right)$ is the signal-to-noise ratio (SNR), $W(\mathrm{~Hz})$ is the bandwidth, $N_{0}$ (Joule) is the noise spectral density and $S=R / W(\mathrm{bit} / \mathrm{s} / \mathrm{Hz})$ is the achievable SE. In addition, $f^{-1}: C \in[0,+\infty) \mapsto \gamma \in$ $[0,+\infty)$ is the inverse function of $f$. For example, $f(\gamma)=$ $\log _{2}(1+\gamma)$ and conversely, $f^{-1}(C)=2^{C}-1$ in the additive white Gaussian noise (AWGN) channel case [2], [3].

Equation (1) clearly indicates that formulating a closedform expression for the EE-SE trade-off of any communication system is equivalent to obtaining an explicit expression for the inverse function of its channel capacity per unit bandwidth, i.e. $f^{-1}(C)$. So far, the latter has only been proved feasible for the 


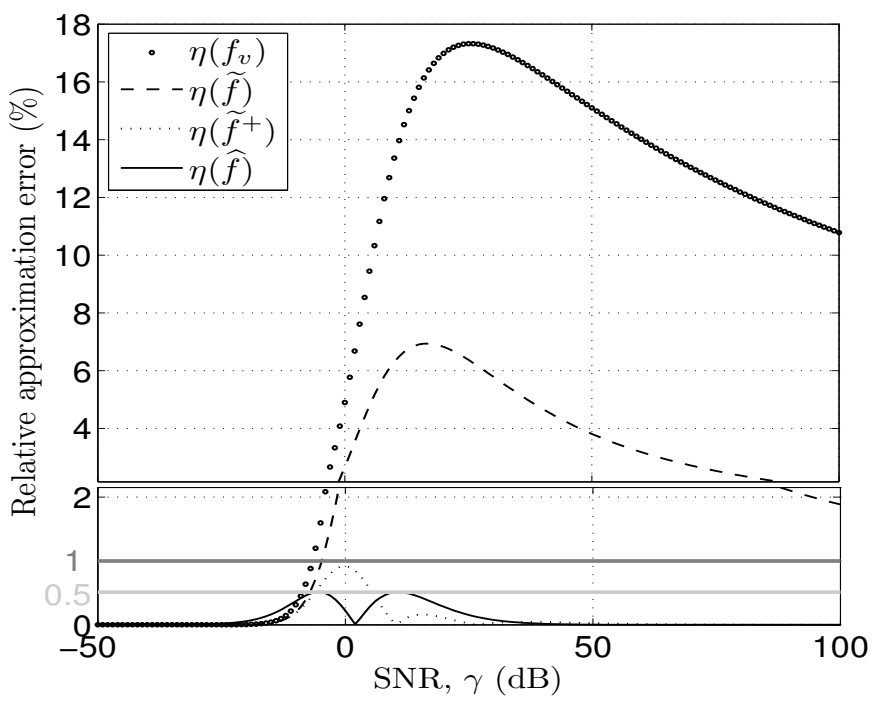

Fig. 1. Relative approximation error between $f$ and $f_{v}, \widetilde{f}, \widetilde{f}^{+}$as well as $\widehat{f}$ vs. $\gamma(\mathrm{dB})$.

AWGN channel and deterministic channel with colored noise in [2] and [3], respectively. Instead, the EE-SE trade-off can easily be formulated in closed-form via an approximation of $f^{-1}(C)$ such for instance as in [3]

$$
f^{-1}(C) \approx f_{v}^{-1}(C)=C\left(E_{b} / N_{0 \min }\right) 2^{\frac{C}{S_{0}}},
$$

and conversely,

$$
f(\gamma) \approx f_{v}(\gamma)=\frac{S_{0}}{\ln (2)} W_{0}\left(\frac{\ln (2) \gamma}{S_{0}\left(E_{b} / N_{0 \text { min }}\right)}\right)
$$

where $W_{0}$ denotes the real branch of the Lambert function [6]. The Lambert $W$ function is the inverse function of $f(w)=$ $w e^{w}$ and, thus, it satisfies $W(z) e^{W(z)}=z$, with $w, z \in \mathbb{C}[6]$. Its real branch, $W_{0}$, is such that $W_{0}: \mathcal{D}_{W_{0}}=\left[-e^{-1},+\infty\right) \mapsto$ $[-1,+\infty)$ and is monotonically increasing over $\mathcal{D}_{W_{0}}$. In addition, $E_{b} / N_{0 \text { min }}=\ln (2) / \dot{f}(0)$ and $S_{0}=-2[\dot{f}(0)]^{2} / \ddot{f}(0)$ are the minimum energy-per-bit and the slope of the SE, respectively, where $\dot{f}(0)$ and $\ddot{f}(0)$ are the first and second order derivatives of $f(\gamma)$ when $\gamma=0$. This method is quite generic, which has made it very popular for approximating the EE-SE trade-off of communication systems in various scenarios [3], [7]-[9]. However, its accuracy is limited to the low-SNR/SE regime, especially in the SISO case, as it can be seen in Fig. 1. In [4] and [10], we have recently proposed an accurate CFA-based approach for formulating the EE-SE trade-off of point-to-point MIMO and cellular uplink systems, respectively, in any SE regime of interest. In our new approach, we have obtained an accurate CFA of $f(\gamma)$, i.e. $f(\gamma) \approx g(\gamma)$, which is invertible and such that $g^{-1}(C)$ has an explicit formulation. In the next section, we extend this approach to the SISO Rayleigh flat fading channel scenario.

\section{IMPROVED CFA OF THE SISO ERGODIC CAPACITY}

Assuming that the number of transmit and receive antennas, $t$ and $r$, respectively, is equal to one in (2.38) of [11], the ergodic capacity per unit bandwidth of the SISO Rayleigh flat fading channel can be formulated as

$$
f(\gamma)=e^{\gamma^{-1}} \mathrm{E}_{1}\left(\gamma^{-1}\right) / \ln (2)
$$

with $E_{1}$ being the exponential integral function. The function $f$ is continuous and differentiable for $\gamma \in[0,+\infty]$ such that $\frac{\partial f}{\partial \gamma}=\frac{1}{\gamma}\left(1-\frac{f(\gamma)}{\gamma}\right)$. Moreover, since $f(\gamma)<\gamma$, it implies that $\frac{\partial f}{\partial \gamma}>0$ and, thus, $f$ is strictly increasing and in turn invertible. However, to the best of our knowledge, an explicit formulation of $f^{-1}$ does not exist. As previously explained, we have recently proposed in [4] a novel approach for deriving a CFA of the MIMO EE-SE trade-off by using the CFA of the MIMO ergodic capacity per unit bandwidth in [12], i.e. $f(\gamma) \approx$

$\tilde{f}(\gamma)=\frac{2 n}{\ln (2)}\left[-\left(\frac{1}{2}+\ln (2)\right)+\frac{1}{1+\sqrt{1+4 \gamma}}+\ln (1+\sqrt{1+4 \gamma})\right]$

when $n=t=r$, as a starting point for our derivation. The advantage of the latter over the exact closed-form expression in [11] is that its inverse can be explicitly derived. However, the CFA of [12] has been derived by using random matrix theory and considering a large number of antenna elements $n$; consequently, this approximation is not very accurate for the case of $n=1$, i.e. SISO case, as it is depicted in Fig. 1. In an attempt to improve the accuracy of $f(\gamma)$, we have recently derived in [5] an improved approximation of $f(\gamma)$ denoted $\widetilde{f}^{+}(\gamma)$ by designing a parametric function that tightly fits $f(\gamma)-\widetilde{f}(\gamma)$. As a result, we have obtained an accurate CFA of $f(\gamma)$ but with a cumbersome expression for its inverse. In order to have both a very tight CFA of $f(\gamma)$ with a simple and accurate formulation for its inverse, we obtain here $f(\gamma) \approx$

$$
\begin{aligned}
\widehat{f}(\gamma) & =\frac{1}{\ln (2)}\left[-\phi+\phi\left(1-\alpha(b, \phi)^{b}+[\gamma+\alpha(b, \varphi)]^{b}\right)^{-1}\right. \\
& \left.+\frac{1}{b} \ln \left(1-\alpha(b, \phi)^{b}+[\gamma+\alpha(b, \varphi)]^{b}\right)\right]
\end{aligned}
$$

where $\alpha(b, \phi)=e^{\frac{1}{1-b} \ln (1-b \phi)}$ and $\phi=0.57721 \ldots$ denotes the Euler-Mascheroni constant [13]. Note that derivation details for obtaining (7) are provided in Section A of the Appendix. In addition, the value of the parameter $b$ that minimizes the maximum of the relative approximation error between $f(\gamma)$ and $\widehat{f}(\gamma)$ has been obtained by solving

$$
\begin{aligned}
& \min _{b} \max _{\gamma}\{\eta(\hat{f})\} \\
& \text { s.t. } \quad 0 \leq b \leq 1 / \phi \text { and }-50 \leq \gamma(\mathrm{dB}) \leq 100,
\end{aligned}
$$

where $\eta(g)=100|f(\gamma)-g(\gamma)| /|f(\gamma)|$, such that $b=0.7066$. In Fig. 1, we plot the relative error between $f$ and $f_{v}, \tilde{f}$, $\widetilde{f}^{+}$as well as $\widehat{f}$, as a function of the SNR $\gamma$. Note that the lower part of the graph (range of relative error from 0 to 2.1 $\%$ ) has been magnified for improving the readability of this graph. Results clearly indicate that our novel CFA $\widehat{f}$ in (7) is the most accurate of the four CFAs, it differs from $f$ at worst by $0.5 \%$ and on average by $0.1 \%$. They also confirm that the method of [3], i.e. $f_{v}$, is only valid at low SNR.

\section{CFA OF THE SISO EE-SE TRADE-OFF}

The main advantage of $\widehat{f}$ over $f$ is the fact that its inverse function $\hat{f}^{-1}$ can be formulated into a simple and accurate closed-form such that $f^{-1}(C) \approx$

$\widehat{f}^{-1}(C)=\left[\alpha(b, \varphi)^{b}-1-b \varphi\left[W_{0}\left(-b \varphi e^{-b \varphi} 2^{-\frac{b C}{n}}\right)\right]^{-1}\right]^{\frac{1}{b}}-\alpha(b, \varphi)$, 
where $\varphi=1+\phi-\left(\sum_{i=1}^{n} \frac{1}{i}-\ln (n)\right)$. Derivation details about (9) are given in Section B of the Appendix. The value of $b$ that minimizes the maximum of the absolute approximation error between $f^{-1}(C)$ and $\widehat{f}^{-1}(C)$ can be obtained by solving

$$
\begin{array}{ll}
\min _{b} & \max _{C}\left\{\epsilon\left(\hat{f}^{-1}\right)\right\} \\
\text { s.t. } & 0 \leq b \leq 1 / \varphi \text { and } 0 \leq C \leq 40,
\end{array}
$$

where $\epsilon(g)=\left|f^{-1}(C)-g(C)\right|$, such that $b=0.71435$, $0.63363,0.6003$ and 0.5815 for $n=1,2,3$ and 4 in (9), respectively. Since $f^{-1}$ does not have a closed-form expression, the nearly-exact $f^{-1}$ has been obtained numerically by using the method described in [4]. In this letter, we aim at deriving an improved CFA of the EE-SE trade-off for the SISO Rayleigh flat fading channel, however, (9) is also an improved CFA for the $n \times n$ MIMO channel, as it is indicated in Fig. 2. We plot in Fig. 2 the absolute error in $\mathrm{dB}$ between $f^{-1}$ and $\widetilde{f}^{-1}$ in (18) of [4] as well as between $f^{-1}$ and $\widehat{f}^{-1}$ in (9) vs. the SE for $n=1,2,3$ and 4. Note that as in Fig. 1, the lower part of the graph (range of absolute error from 0 to $0.11 \mathrm{~dB}$ ) has been magnified. In the SISO case, results indicate that (9) allows us to reduce the maximum of the approximation error from $1.8 \mathrm{~dB}$ to $0.05 \mathrm{~dB}$ in comparison with the CFA in (18) of [4], which graphically confirms the high accuracy of (9). Moreover, the approximation error of (9) is always lower than (18) of [4] for any SE value. In the $n \times n$ MIMO scenario, (9) provides a lower approximation error than (18) of [4], i.e. $\epsilon\left(\widehat{f}^{-1}\right)<0.11$ $\mathrm{dB}$ whereas $\max \left\{\epsilon\left(\tilde{f}^{-1}\right)\right\}=1,0.6$ and 0.4 for $n=2,3$ and 4 , respectively. Contrary to the SISO case, it can be noted that (9) is less accurate than (18) of [4] in the low-SE regime but far more accurate otherwise, especially for $n=2$. However, as $n$ increases as the error reduction gain of (9) over (18) of [4] diminishes up to a point where (18) of [4] will become more accurate than (9) on average. As it is explained in the Appendix, $f$ and $\widetilde{f}$ differ by $|\varphi-1|=\phi-\left(\sum_{i=1}^{n} \frac{1}{i}-\ln (n)\right)$ at high SNR. Since $\phi=\lim _{m \rightarrow+\infty}\left(\sum_{i=1}^{m} \frac{1}{i}-\ln (m)\right)$, it implies that $|\varphi-1|$ converges towards zero as $n$ increases and, hence, the accuracy of $\widetilde{f}$ increases. Thus, we can conclude that (9) should mainly be used for accurately approximating $f^{-1}(C)$ for $n=1$ up to 5 and, then, it is better to use (18) of [4] for higher values of $n$.

\section{CONCLUSION}

In this letter, a very tight CFA of the EE-SE trade-off over the SISO Rayleigh flat fading channel has been derived. First we have proposed an improved approximation of the SISO ergodic capacity and then utilized this approximation to derive our CFA, which has been generalized for the symmetric MIMO channel. The great accuracy of our novel CFA has been numerically shown for a wide range of SE values, especially in comparison with the existing approximations.

\section{APPENDIX}

\section{A. Derivation Insights: How to obtain (7)}

Knowing that $\mathrm{E}_{1}(x) \stackrel{x \rightarrow+\infty}{\sim} \frac{e^{-x}}{x}$, it implies that $f(\gamma)$ in (5) simplifies as

$$
f(\gamma) \stackrel{\gamma \rightarrow 0}{\sim} f_{0}(\gamma)=\frac{\gamma}{\ln (2)}
$$



Fig. 2. Absolute approximation error between $f^{-1}$ and $\widetilde{f}^{-1}$ as well as $f^{-1}$ and $\widehat{f}^{-1}$ vs. SE (bit/s/Hz).

at low SNR. Similarly, $f(\gamma)$ simplifies as

$$
f(\gamma) \stackrel{\gamma \rightarrow+\infty}{\sim} \frac{-\phi+\ln (\gamma)+\frac{1}{\gamma}(1-\phi+\ln (\gamma))+\frac{1}{\gamma^{2}}}{\ln (2)}
$$

at high SNR, since $e^{x} \stackrel{x \rightarrow 0}{\sim} 1+x$ and $\mathrm{E}_{1}(x) \stackrel{x \rightarrow 0}{\sim}-\phi-\ln (x)+x$. The latter equation further simplifies as

$$
f(\gamma) \stackrel{\gamma \rightarrow+\infty}{\sim} f_{\infty}(\gamma)=\frac{-\phi}{\ln (2)}+\log _{2}(\gamma)
$$

in the SISO case. Moreover, it can easily be proved that $f_{0}(\gamma)$ and $f_{\infty}(\gamma)$ can be generalized as

$$
f_{0}(\gamma)=\frac{n \gamma}{\ln (2)} \text { and } f_{\infty}(\gamma)=n\left(\frac{-\varphi}{\ln (2)}+\log _{2}(\gamma)\right)
$$

in the $n \times n$ MIMO case by using (2.38) of [11], where $\varphi=$ $1+\phi-\left(\sum_{i=1}^{n} \frac{1}{i}-\ln (n)\right)$.

As far as $\tilde{f}(\gamma)$ in (6) is concerned, $\sqrt{1+4 \gamma}=e^{0.5 \ln (1+4 \gamma)}$ simplifies as $1+2 \gamma$ at low SNR, which in turn implies that

$$
\widetilde{f}(\gamma) \stackrel{\gamma \rightarrow 0}{\sim} \frac{2 n}{\ln (2)}\left[-\frac{1}{2}+\frac{1}{2}(1-\gamma)+\ln (1+\gamma)\right]
$$

since $(1+x)^{-1} \stackrel{x \rightarrow 0}{\sim} 1-x$. Consequently, $\widetilde{f}(\gamma)$ is equivalent to

$$
\widetilde{f}(\gamma) \stackrel{\gamma \rightarrow 0}{\sim} \widetilde{f}_{0}(\gamma)=\frac{n \gamma}{\ln (2)}
$$

at low SNR. Similarly, $\sqrt{1+4 \gamma}$ simplifies as $2 \sqrt{\gamma}$ at high SNR such that

$$
\begin{aligned}
& \tilde{f}(\gamma) \stackrel{\gamma \rightarrow+\infty}{\sim} \frac{2 n}{\ln (2)}\left[-\frac{1}{2}+\frac{1}{2 \sqrt{\gamma}}+\ln (\sqrt{\gamma})\right], \\
& \stackrel{\gamma \rightarrow+\infty}{\sim} \widetilde{f}_{\infty}(\gamma)=n\left(\frac{-1}{\ln (2)}+\log _{2}(\gamma)\right) .
\end{aligned}
$$

It can be seen from equations (14) and (16) that $f(\gamma)$ and $\tilde{f}(\gamma)$ are equivalent in the low-SNR regime. Whereas, in the high-SNR regime, equations (14) and (17) reveal that $f(\gamma)$ and $\widetilde{f}(\gamma)$ differ by $|\varphi-1|$, which explains the shape of the 
curve of $\eta(\widetilde{f})$ in Fig. 1. In order to obtain an improved version of $\widetilde{f}(\gamma)$, we design a parametric function $\widehat{f}(\gamma)$ based on $\widetilde{f}(\gamma)$ in (6) but that ensures that $\widehat{f}(\gamma)$ is equivalent to $f(\gamma)$ both at low and high SNRs such that

$$
\widehat{f}(\gamma)=\frac{n}{b \ln (2)}\left[-\frac{1}{B}+\frac{1}{A(\gamma)}-\ln (B)+\ln (A(\gamma))\right],
$$

with $A(\gamma)=d+e^{b \ln (c+a \gamma)}$. Note that $\widehat{f}(\gamma)=\tilde{f}(\gamma)$ for $a=4$, $B=2, c=d=1$ and $b=0.5$ in (18). In the low-SNR regime, $A(\gamma)=d+e^{b \ln (c+a \gamma)}=d+e^{b \ln \left(c\left(1+\frac{a}{c} \gamma\right)\right)}$ simplifies as

$$
\begin{aligned}
A(\gamma) & \stackrel{\gamma \rightarrow 0}{\sim} d+e^{b \ln (c)}\left(1+\frac{b a}{c} \gamma\right) \\
& \gamma \rightarrow 0 \\
& \sim\left(d+e^{b \ln (c)}\right)\left(1+\frac{b a e^{b \ln (c)}}{c\left(d+e^{b \ln (c)}\right)} \gamma\right) .
\end{aligned}
$$

Consequently, we obtain by inserting (19) into (18) that

$$
\begin{gathered}
\widehat{f}(\gamma) \stackrel{\gamma \rightarrow 0}{\sim} \frac{n}{b \ln (2)}\left[-\frac{1}{B}+\frac{1}{d+e^{b \ln (c)}}\left(1-\frac{b a e^{b \ln (c)}}{c\left(d+e^{b \ln (c)}\right)} \gamma\right),\right. \\
\left.-\ln (B)+\ln \left(d+e^{b \ln (c)}\right)+\frac{b a e^{b \ln (c)}}{c\left(d+e^{b \ln (c)}\right)} \gamma\right]
\end{gathered}
$$

since $(1+u x)^{-1} \stackrel{x \rightarrow 0}{\sim}(1-u x)$ and $\ln (1+u x) \stackrel{x \rightarrow 0}{\sim} u x$. Let us assume that $B=d+e^{b \ln (c)}$ in (20), then the latter further simplifies as

$$
\widehat{f}(\gamma) \stackrel{\gamma \rightarrow 0}{\sim} \widehat{f}_{0}(\gamma)=\left[\left(1-\frac{1}{B}\right) \frac{b a e^{b \ln (c)}}{c B}\right] \frac{n \gamma}{b \ln (2)} .
$$

Moreover, we obtain the following relation

$$
c=e^{\frac{1}{1-b} \ln \left(\frac{a(B-1)}{B^{2}}\right)}
$$

by enforcing $f_{0}(\gamma)=\widehat{f}_{0}(\gamma)$. Similarly, $A(\gamma) \stackrel{\gamma \rightarrow+\infty}{\sim} e^{b \ln (a \gamma)}$ at high SNR and, hence, $A^{-1}(\gamma) \stackrel{\gamma \rightarrow+\infty}{\sim} 0$ as well as $\ln (A(\gamma)) \stackrel{\gamma \rightarrow+\infty}{\sim} b \ln (a \gamma)$. Thus, $\widehat{f}(\gamma)$ in (18) can be reexpressed as

$$
\widehat{f}(\gamma) \stackrel{\gamma \rightarrow+\infty}{\sim} \widehat{f}_{\infty}(\gamma)=\frac{-n}{B b \ln (2)}-\frac{n}{b} \log _{2}(B)+n \log _{2}(a \gamma)
$$

Then, we obtain that

$$
B=d+e^{b \ln (c)}=(b \varphi)^{-1}
$$

by ensuring that $f_{\infty}(\gamma)=\widehat{f}_{\infty}(\gamma)$, which in turn implies that

$$
d=(b \varphi)^{-1}-e^{b \ln (c)} .
$$

In addition, the equality $\log _{2}(\gamma)=-\frac{1}{b} \log _{2}(B)+\log _{2}(a \gamma)$ indicates that

$$
a=e^{\frac{1}{b} \ln (B)} .
$$

Using equations (22), (24), (25) and (26), we can re-express the parameters $B, a, c$ and $d$ solely as a function of $\varphi$ and the parameter $b$, as follows,

$$
\left\{\begin{array}{l}
B=(b \varphi)^{-1} \\
a=e^{-\frac{1}{b} \ln (b \varphi)} \\
c=e^{-\frac{1}{b} \ln (b \varphi)} e^{-\frac{1}{1-b} \ln (1-b \varphi)} \\
d=(b \varphi)^{-1}\left(1-e^{-\frac{b}{1-b} \ln (1-b \varphi)}\right) .
\end{array}\right.
$$

Inserting (27) into (18), we can re-express our parametric function as

$$
\begin{aligned}
\widehat{f}(\gamma) & =\frac{n}{\ln (2)}\left[-\varphi+\varphi\left(1-\alpha(b, \varphi)^{b}+[\gamma+\alpha(b, \varphi)]^{b}\right)^{-1}\right. \\
& \left.+\frac{1}{b} \ln \left(1-\alpha(b, \varphi)^{b}+[\gamma+\alpha(b, \varphi)]^{b}\right)\right]
\end{aligned}
$$

where $\alpha(b, \varphi)=e^{\frac{1}{1-b} \ln (1-b \varphi)}$, which revert to (7) in the SISO case, i.e. $n=1$.

\section{B. Derivation Insights: How to obtain (9) from (28)}

We know that $C=f(\gamma) \approx \widehat{f}(\gamma)$ and, hence, it implies with (28) that

$$
C \ln (2) \approx n\left[-\varphi+\varphi Z^{-1}+\frac{1}{b} \ln (Z)\right],
$$

where $Z=1-\alpha(b, \varphi)^{b}+[\gamma+\alpha(b, \varphi)]^{b}$. Equivalently, we can re-expressed (29) as

$$
\begin{array}{rlrl}
-b(C \ln (2) / n+\varphi) & \approx-b \varphi Z^{-1}-\ln (Z), \\
\Leftrightarrow-b \varphi e^{-b\left(\frac{C \ln (2)}{n}+\varphi\right)} & \approx-b \varphi Z^{-1} e^{-b \varphi Z^{-1},} \\
\Leftrightarrow & Z^{-1} & \approx-(b \varphi)^{-1} W_{0}\left(-b \varphi e^{-b\left(\frac{C \ln (2)}{n}+\varphi\right)}\right), \\
\Leftrightarrow & Z & \approx-b \varphi\left[W_{0}\left(-b \varphi e^{-b \varphi} 2^{-\frac{b C}{n}}\right)\right]^{-1},
\end{array}
$$

which is finally equivalent to (9) after further simplifications.

\section{REFERENCES}

[1] G. Auer et al., "How Much Energy is Needed to Run a Wireless Network ?" IEEE Wireless Commun. Mag., vol. 18, no. 5, pp. 40-49, Oct. 2011.

[2] H. M. Kwon and T. G. Birdsall, "Channel Capacity in Bits per Joule," IEEE J. Ocean. Eng., vol. OE-11, no. 1, pp. 97-99, Jan. 1986.

[3] S. Verdu, "Spectral Efficiency in the Wideband Regime," IEEE Trans. Inf. Theory, vol. 48, no. 6, pp. 1319-1343, Jun. 2002.

[4] F. Héliot, M. A. Imran, and R. Tafazolli, "On the Energy EfficiencySpectral Efficiency Trade-off over the MIMO Rayleigh Fading Channel," IEEE Trans. Commun., vol. 60, no. 5, pp. 1345-1356, May 2012.

[5] —, "A Tight Closed-Form Approximation of the SISO Energy Efficiency-Spectral Efficiency Trade-Off," in Proc. ICT Future Network \& Mobile Summit, Berlin, Germany, Jul. 2012.

[6] R. M. Corless, G. H. Gonnet, D. E. G. Hare, D. J. Jeffrey, and D. E. Knuth, "On the LambertW Function," Adv. Comput. Math., vol. 5, pp. 329-359, 1996.

[7] A. Lozano, A. M. Tulino, and S. Verdu, "Multiple-antenna Capacity in the Low-power Regime," IEEE Trans. Inf. Theory, vol. 49, no. 10, pp. 2527-2544, Oct. 2003.

[8] G. Caire, G. Taricco, and E. Biglieri, "Suboptimality of TDMA in the Low-power Regime," IEEE Trans. Inf. Theory, vol. 50, no. 4, pp. 608620, Apr. 2004.

[9] Y. Yao, X. Cai, and G. B. Giannakis, "On Energy Efficiency and Optimum Resource Allocation of Relay Transmissions in the Low-power Regime," IEEE Trans. Wireless Commun., vol. 4, no. 6, pp. 2917-2927, Nov. 2005.

[10] O. Onireti, F. Héliot, and M. A. Imran, "On the Energy EfficiencySpectral Efficiency Trade-Off in the Uplink of CoMP System," IEEE Trans. Wireless Commun., vol. 11, no. 2, pp. 556-561, Feb. 2012.

[11] M. Dohler, "Virtual Antenna Arrays," Ph.D. dissertation, King's College London, University of London, Nov. 2003.

[12] E. Biglieri and G. Taricco, Transmission and Reception with Multiple Antennas: Theoretical Foundations. Now Publishers Inc., 2004.

[13] X. Gourdon and P. Sebah, "The Euler constant: $\gamma$." [Online]. Available: http://numbers.computation.free.fr/Constants/Gamma/gamma.html 